\title{
Prevalencia del catastro sobre el Registro de Predios PARA CORREGIR INEXACTITUdeS REGISTRALES
}

\author{
Guillermo García Montúfar Sarmiento* \\ Universidad de Lima, Lima, Perú \\ ggarciam@ldgabogados.com \\ FREDY LUIS SiLVA VILLAJUÁN** \\ Universidad Nacional Mayor de San Marcos, Lima, Perú \\ fsilva@ldgabogados.com
}

Recibido: 23/11/2020 Aceptado: 30/11/2020

doi: https://doi.org/10.26439/iusetpraxis2021.n052.5219

\begin{abstract}
RESUMEN. El catastro, entendido como el inventario físico de los predios que conforman el territorio nacional, es de especial importancia para las inmatriculaciones de predios, puesto que, entre otras funciones, permite resolver discrepancias entre el registro y las dimensiones reales de los predios, y armonizar la verdad física con la realidad jurídica. Sin embargo, en la realidad, la aplicación del sistema catastral ha sido difícil, puesto que existen pocas municipalidades declaradas como zonas catastrales en el Perú y, por lo tanto, no ha sido posible vincular al Sistema Nacional Integrado de Información Catastral Predial (SNCP) con el Registro de Predios. Entonces, la función del catastro se ha limitado a la rectificación de áreas de predios mediante procesos de saneamiento registral. Por otro lado, en los casos en que la discrepancia entre la información catastral y la registral está dentro de los límites de tolerancia, prevalece la información del catastro, mediante título emitido por ente generador de catastro y sin afectar los derechos de terrenos colindantes.
\end{abstract}

PALABRAS CLAVE: catastro / inmatriculación / registro / predio / inmueble / titularidad / descripción física

\footnotetext{
* Magíster en Derecho Civil y Comercial, con estudios de especialización en las Facultades de Derecho de las Universidades de Wisconsin y de Harvard, Estados Unidos. Profesor de Derecho Civil de la Universidad de Lima.

** Abogado por la Universidad Nacional Mayor de San Marcos, con estudios de especialización en Derecho Registral en la Universidad Ramon Llull, Barcelona, España. Fue registrador público, vocal y presidente del Tribunal Registral de la Sunarp.
} 


\title{
PREVALENCE OF CADASTRE OVER THE REAL ESTATE REGISTRY TO FIX MISCALCULATIONS IN THE REGISTRY
}

\begin{abstract}
Cadastre, understood as the compilation of al cadastral maps within the territory of this country, is especially important for registration of real estate, because it allows to solve discrepancias between the information in the registry and the real dimensions of land, harmonizing the physical reality with the legal truth. However, in practice it has been difficult to apply the cadastral system, because there are few municipalities declared as cadastral zones in Peru, therefore it has not been posible to integrate the "sistema nacional integrado de información catastral predial (SNCP)" with the real estate registry, and the role of cadastre has been limited to rectifying the area of land through corrective proccesses. On the other hand, whenever the discrepancies between cadastral information and the registry exist within the tolerated limits, cadastre should prevail, through a title produced by a cadastre generating entity and without affecting the rights over surrounding lands.
\end{abstract}

KEYWORDS: cadastre / registration/ registry / estate / property / ownership / physical description 


\section{INTRODUCCIÓN}

Desde la creación del Registro de la Propiedad Inmueble el 2 de enero de 1888 hasta la promulgación del Decreto Supremo N. ${ }^{\circ}$ 002-89-JUS el 27 de enero de 1989, las inscripciones de las inmatriculaciones o primeras de dominio de los predios, así como sus acumulaciones o subdivisiones, se efectuaban sin soporte de información gráfica catastral. Es decir, la descripción física de los predios se efectuaba únicamente de manera literal y, en algunos casos, acompañada de planos elaborados por los otorgantes del título, sin que dicha descripción física del predio tenga correlato o concordancia con una representación gráfica avalada por un ente oficial generador de catastro.

No queremos decir con esto que a partir de la expedición del Decreto Supremo $\mathrm{N} .^{\circ}$ 002-89-JUS fue obligatoria en todos los casos la presentación de los planos catastrales para la inscripción de los actos de inmatriculación, acumulación o subdivisión de predios, pues si el predio se encontraba ubicado en una zona no catastrada, en vez del plano catastral, debía presentarse la constancia negativa de catastro a fin de posibilitar la inscripción del título'. Sin embargo, sí debemos resaltar que esta norma fue el punto de partida para tratar de vincular el catastro municipal con el Registro de Predios, finalidad que hasta ahora no se ha cumplido a cabalidad.

La inexistencia del catastro, como sustento para las inscripciones de las inmatriculaciones de predios o de los actos que modifiquen su descripción física, trajo como consecuencia innumerables casos de doble y hasta triple inmatriculación. Esto significa que una misma porción del territorio nacional fuese registrada en más de una partida registral, situación que vulnera el principio de especialidad que se plasma en el sistema de inscripción de folio real en el Registro de Predios, según el cual, por cada predio, solamente puede haber una partida registral abierta. Además, afecta la seguridad jurídica, pues, al haber más de una partida registral abierta respecto a un mismo predio, publicitando en la mayoría de los casos titularidades incompatibles, existirá un innegable conflicto para determinar cuál será la titularidad que deberá prevalecer. En este supuesto, no habrá certeza para los operadores jurídicos sobre quién será el titular del derecho con el que se deberá contratar.

Otra consecuencia de la falta de representación gráfica avalada por un ente generador de catastro es la discordancia que existe entre la descripción literal del predio que consta en la partida registral -y en los respectivos títulos archivados que sustentaron dichas inscripciones- y la descripción física real del predio. Para ello, basta con efectuar el levantamiento topográfico del predio con las técnicas de medición actuales y compararlo con la descripción literal que consta en la partida registral para comprobar

1 Tal como actualmente se regula el tema en el primer párrafo del artículo 20 del Reglamento de Inscripciones del Registro de Predios. 
que muchas veces existen discrepancias en el área, linderos, medidas perimétricas y hasta en la ubicación espacial del predio.

Desde luego, la situación antes descrita no ocasiona mayor problema para la calificación e inscripción de los actos o derechos que no impliquen la modificación de la descripción física del predio, pues será suficiente referirse en el título traslativo de dominio o de gravamen a los datos registrales para que este pueda inscribirse en el Registro de Predios sin mayor dificultad, más aún cuando en la mayoría de los contratos de compraventa se incorpora la cláusula ad corpus ${ }^{2}$. Por tanto, en el caso de los predios inscritos, si bien las personas contratan de acuerdo con la información oficial que proviene de las partidas registrales, adquieren el predio tal cual existe en la realidad, independientemente de su descripción física que consta en las partidas registrales.

La discordancia entre la realidad y el registro respecto a la descripción física del predio tiene relevancia cuando se solicite la inscripción de algún acto que modifique su descripción física. Esto suele ocurrir con la inscripción de la parcelación o independización de un predio rústico, habilitación urbana, acumulación, subdivisión, inscripción de una declaratoria de edificación, entre otros actos, en los cuales el registrador, asistido por el Área de Catastro de las Oficinas Registrales ${ }^{3}$, debe evaluar la adecuación del título presentado con los antecedentes registrales del área, linderos, medidas perimétricas y ubicación espacial. En estos casos, si el título presentado es discrepante con sus antecedentes registrales en cuanto a los datos descriptivos del predio, el registrador denegará la inscripción si las discrepancias en el área se encuentran fuera de los rangos de tolerancia o cuando las medidas perimétricas no concuerdan con los datos que constan en los antecedentes registrales, aunque el área se encuentre dentro de dichos rangos ${ }^{4}$.

Es común encontrar observaciones registrales cuando la confrontación del título presentado y sus antecedentes registrales no puede efectuarse debido a que (i) no existe información gráfica del polígono y la ubicación del predio en los antecedentes registrales;

2 Artículo 1577 del Código Civil: "Si el bien se vende fijando precio por el todo y no con arreglo a su extensión o cabida, aun cuando esta se indique en el contrato, el comprador debe pagar la totalidad del precio, a pesar de que se compruebe que la extensión o cabida real es diferente.

Sin embargo, si se indicó en el contrato la extensión o cabida, y la real difiere de la señalada en más de una décima parte, el precio sufrirá la reducción o el aumento proporcional".

3 El Área de Catastro no es un ente generador de catastro, sino una oficina del Registro de Predios que se encarga de acopiar la información gráfica que consta en los títulos inscritos, elaborar a partir de ellos la Base Gráfica Registral y brindar información técnica a los registradores en los procedimientos de inscripción de títulos y en los de publicidad registral, como es la expedición del certificado de búsqueda catastral.

4 Si bien el artículo literal e) del numeral 7 de la Directiva N. ${ }^{\circ} 01-2008-S N C P / C N C$ establece que las tolerancias catastrales y registrales también se aplican cuando se presenten variaciones en las medidas perimétricas y/o perímetro del predio, siempre que el área no exceda los rangos establecidos para las discrepancias de áreas, esta norma en la mayoría de los casos no es aplicada o es aplicada de manera incorrecta en la calificación registral de un título. 
o (ii) existe información gráfica del polígono y la ubicación del predio, pero esta es incompleta o inexacta. El tema es particularmente sensible cuando se solicita la inscripción de la rectificación del área del predio por error de cálculo, o bien la rectificación del área, linderos y medidas perimétricas de manera unilateral. En estos supuestos, conforme ha establecido el Tribunal Registral de la Sunarp (en adelante, TR) en sendos precedentes de observancia obligatoria ${ }^{5}$, se requiere, además de los planos visados por la municipalidad respectiva, el informe favorable del Área de Catastro del Registro afirmando que existe concordancia en cuanto a los linderos, medidas perimétricas y ubicación espacial del predio (en el caso de la rectificación de área por error de cálculo), o que el polígono del predio de menor extensión se encuentra dentro del polígono del predio de mayor extensión conforme a su descripción registral (en la rectificación unilateral). No podrán aplicarse estos precedentes de observancia obligatoria si en los antecedentes registrales no existe documentación gráfica que permita al Área de Catastro del Registro comparar el polígono registral del predio con el polígono real que consta en el plano visado por la municipalidad respectiva. Tampoco se aplicarán cuando el Área de Catastro del Registro haya incorporado de manera incorrecta el polígono del predio a la Base Gráfica Registral (en adelante, BGR) ${ }^{6}$ y, como consecuencia de ello, se genere un desfase con el polígono real del predio.

En el caso de que las discrepancias entre la descripción física real de los predios y la descripción registral superen los rangos de tolerancias catastrales y registrales ${ }^{7}$, deberá recurrirse a alguno de los procedimientos de rectificación de área previstos en la normativa vigente ${ }^{8}$.

El objeto del presente artículo es sustentar la prevalencia de la información catastral sobre la información registral para corregir las inexactitudes registrales sin tener que recurrir a los procedimientos de rectificación de área, en los supuestos de inexistencia,

5 En la tercera sección del presente artículo, nos referiremos con mayor detalle a los precedentes de observancia obligatoria sobre rectificación de área por error de cálculo y de manera unilateral.

6 El numeral 5.1 de la Directiva N. ${ }^{\circ}$ 002-2014/SUNARP-SN define a la BGR como el "[...] sistema de información registral estructurada y organizada, constituida por la base de datos gráficos y alfanuméricos automatizados de predios inscritos en el Registro de Predios, a partir de la información técnica que obra en los títulos archivados, elaborado sobre una Cartografía Base".

7 El literal a) del artículo 7 de la Directiva N. ${ }^{\circ}$ 01-2008-SNCP/CNC establece los rangos de tolerancias para predios de naturaleza urbana y rural.

8 La normativa vigente establece que la rectificación de área, linderos y medidas perimétricas de un predio puede efectuarse de las siguientes maneras: (i) la rectificación de área por mutuo acuerdo y por escritura pública; (ii) el procedimiento notarial no contencioso; (iii) el procedimiento de saneamiento catastral registral; y (iv) el proceso judicial de rectificación de área. Resaltamos que el procedimiento notarial no contencioso es aplicable solamente para los predios urbanos o ubicados en zona urbana, en tanto que los demás procedimientos son aplicables para todo tipo de predios. En el caso de los predios rurales, también resulta aplicable el procedimiento especial previsto en el artículo 79 y siguientes del Decreto Supremo N. ${ }^{\circ}$ 032-2008-VIVIENDA. 
deficiencia o error en la información gráfica que obra en los antecedentes registrales, siempre que las discrepancias en cuanto a las áreas se encuentren dentro de los rangos de tolerancia establecidos.

\section{DE LOS ENTES GENERADORES DE CATASTRO Y SU ROL EN LA VERIFICACIÓN DE LA DESCRIPCIÓN FÍSICA DE LOS PREDIOS}

Sabemos que el catastro y el Registro de Predios persiguen fines distintos, pues el primero contiene la descripción de los predios, que incluye sus características físicas, económicas, uso, infraestructura y equipamiento, con fines principalmente tributarios y de planeamiento territorial, mientras que el Registro publicita los derechos que recaen sobre dichos predios. Sin embargo, ambos están íntimamente vinculados, pues como señala Cano Tello (1992):

La coordinación entre el catastro y el Registro es muy necesaria por cuanto que uno incide sobre la realidad física y el otro sobre la realidad jurídica de las fincas. El catastro puede y debe ser un instrumento precioso para la identificación de los inmuebles que son objeto de las relaciones jurídicas inscribibles. (p. 182)

Por esta razón, mediante la Ley N. ${ }^{\circ} 28294$, de fecha 20 de julio del 2004, se crea el Sistema Nacional Integrado de Información Catastral Predial (SNCP), con la finalidad de regular la integración y unificación de los estándares, nomenclatura y procesos técnicos de las diferentes entidades generadoras de catastro en el país y vincularlos con el Registro de Predios.

La vinculación del SNCP con el Registro de Predios se describe en el artículo 5 del Reglamento de la Ley N. ${ }^{\circ} 28294$, aprobado por Decreto Supremo N. ${ }^{\circ}$ 005-2006-JUS (en adelante, Reglamento de la Ley $\mathrm{N}^{\circ}$ 28294), como aquel mecanismo que permite que la información catastral de un determinado predio se relacione con su partida registral, a fin de contar con información integrada de los derechos sobre los predios inscritos en la Superintendencia Nacional de los Registros Públicos (Sunarp), sus características económicas y la visualización de sus características físicas.

El elemento identificatorio que permitirá vincular un predio inscrito en el Registro de Predios con el SNCP es el Código Único Catastral (CUC) , el cual, una vez emitido por la entidad generadora de catastro, debe inscribirse en la partida registral del predio. Es más, de acuerdo con el primer párrafo del artículo 21 de la Ley $N .^{\circ} 28294$, para inscribir de manera definitiva cualquier derecho en el Registro de Predios, debe exigirse la previa inscripción del CUC. En caso de no contar con dicho documento, debería procederse

9 El CUC es definido por el primer párrafo del numeral 3 del artículo 14 de la Ley N. ${ }^{\circ} 28294$, como la identificación alfanumérica de predios. 
únicamente con la anotación preventiva del acto o derecho hasta que se presente el CUC del predio. De haberse aplicado esta norma, hubiera significado que casi la universalidad de los títulos que se presentan al Registro de Predios se tendrían que haber anotado preventivamente, pues incluso ahora son muy pocas las municipalidades que han sido declaradas como zonas catastradas ${ }^{10}$ y que, por ende, están en posibilidad de otorgar el CUC de un predio. En este sentido, a pesar de haber transcurrido más de catorce años desde que se emitió el Reglamento de la Ley N. ${ }^{\circ} 28294$, no se ha logrado implementar la vinculación efectiva del SNCP con el Registro de Predios ${ }^{11}$. Así pues, actualmente el CUC, lejos de servir como elemento de conexión entre la partida registral y el catastro, es utilizado principalmente como un mecanismo para la rectificación del área, linderos y medidas perimétricas de un predio a través del procedimiento de saneamiento catastral registral.

No obstante la situación antes descrita, no podemos dejar de reconocer la finalidad de la Ley N.² 28294, que persigue la integración y unificación de los estándares, nomenclatura y procesos técnicos de las diferentes entidades generadoras de catastro en el país, aunque uno de sus fines más importantes - que es la vinculación del catastro con el Registro de Predios a través de la inscripción el CUC— no se haya cumplido. Sin embargo, para los efectos del presente artículo, queremos rescatar la finalidad de los entes generadores de catastro, principalmente las municipalidades distritales, como entidades que deberían proporcionar información oficial fehaciente respecto a la descripción gráfica del área, linderos, medidas perimétricas y ubicación espacial de un predio.

Así tenemos que, de conformidad con lo previsto por el numeral 3.3 del artículo 79 de la Ley N. ${ }^{\circ} 27972$, Ley Orgánica de Municipalidades, es facultad exclusiva de las municipalidades distritales elaborar y mantener el catastro. En la misma línea, el artículo 15 de la Ley N. ${ }^{\circ} 28294$ precisa que las municipalidades son los organismos generadores de catastro y se interconectan con el Registro de Predios enviándoles la información gráfica y alfanumérica de cada predio para la inscripción del CUC.

10 Según la información de la que disponemos, a la fecha solamente han sido declaradas como zonas catastradas los distritos de Miraflores, San Isidro y el Cercado de Lima, así como algunos sectores del distrito de Los Olivos.

11 La principal razón que impide que se haga efectiva la vinculación del catastro con el Registro es que las municipalidades no hacen levantamiento catastral o no actualizan el levantamiento realizado. Así, según información del Registro Nacional de Municipalidades del 2017, elaborado por el INEl y citado por el diario El Comercio en su edición de fecha 11 de febrero del 2019: “[...] de las 1851 municipalidades provinciales y distritales, solo el $55,2 \%$ ha realizado alguna vez un levantamiento catastral. Sin embargo, de aquellos que han levantado catastro solo el $20,2 \%$ ha realizado una actualización del mismo. Según antigüedad, un cuarto de las municipalidades realizó el último registro catastral hace más de diez años. Además, se estima que las municipalidades que actualizaron este registro entre 2013-2017 se demoraron, en promedio, seis años". 
Por su parte, el numeral 4 del artículo 14 de la Ley N. ${ }^{\circ} 28294$ define al catastro de predios de esta manera:

[...] el inventario físico de los predios orientado a un uso multipropósito, y se encuentra constituido por la suma de predios contiguos que conforman el territorio de la República, a los cuales se les asigna un Código Único Catastral con referencia al titular o titulares del derecho de propiedad del predio. [...].

Asimismo, el literal e) del artículo 3 del Reglamento de la Ley N. ${ }^{\circ} 28294$ ha precisado que el catastro de predios es el inventario físico de todos los predios que conforman el territorio nacional, incluyendo sus características físicas, económicas, uso, infraestructura, equipamiento y derechos inscritos o no en el Registro de Predios.

Queda claro, entonces, que en nuestro sistema jurídico el catastro de predios es elaborado y actualizado principalmente por las municipalidades distritales a nivel nacional. De tal manera, son estas entidades las que tienen competencia exclusiva y excluyente para establecer de manera oficial cuáles son las dimensiones y la ubicación espacial de un predio, salvo, claro está, los supuestos especiales en que dicha competencia ha sido otorgada a alguna entidad encargada de la formalización de la propiedad predial. Por ello, el literal b) del artículo 505 del Código Procesal Civil establece que uno de los requisitos de la demanda de rectificación de área, linderos y medidas perimétricas de un predio es que se presente un plano de ubicación y perimétrico visado por la autoridad municipal o administrativa correspondiente.

Los artículos 60 y siguientes del Reglamento de la Ley N. ${ }^{\circ} 28294$ regulan el procedimiento de saneamiento catastral registral para rectificar el área, linderos y medidas perimétricas de un predio en mérito del CUC.

Si bien a la fecha no es viable obtener un CUC en la mayoría de las municipalidades del país, debido a que no han sido reconocidas como zonas catastradas (situación que impide seguir el procedimiento de saneamiento catastral registral que, como hemos señalado, permite rectificar el área, linderos y medidas perimétricas de un predio y vincular el Registro de Predios con el catastro municipal conforme a la finalidad de la Ley $\mathrm{N}^{\circ}$ 28294), no debe perderse de vista que todas las municipalidades a nivel nacional tienen competencia para elaborar y mantener su respectivo catastro por mandato de la Ley Orgánica de Municipalidades. Desde esta perspectiva, están obligadas, previa inspección ocular del predio, a visar los planos que contengan la representación física de los predios, aunque no cumplan con los requisitos para emitir el CUC. En este sentido, los planos visados por la municipalidad cumplen con el propósito de recoger la realidad física de los predios y pueden servir para corregir inexactitudes registrales en los supuestos en que no se afecte derechos de los propietarios de los predios colindantes. 


\section{DE LA PREVALENCIA DE LA INFORMACIÓN CATASTRAL SOBRE LA REGISTRAL}

Como hemos señalado en la introducción del presente artículo, la mayoría de las inscripciones de las inmatriculaciones o de los actos posteriores que modifican la descripción física de los predios se han realizado, y continúan realizándose, sin que tengan un correlato en información gráfica validada por los entes generadores de catastro. Ello ha ocasionado que en un alto porcentaje de inscripciones de predios exista un divorcio entre la descripción literal que emana de las partidas registrales (y sus respectivos títulos archivados) y la descripción física real de un predio. Esta situación conduce a la denegatoria de la solicitud de inscripción de los títulos referidos a la modificación de la descripción física del predio, por la inadecuación con sus antecedentes registrales.

La normativa vigente ha establecido que, cuando las discrepancias se encuentren fuera de los rangos de tolerancia, deberá seguirse alguno de los procedimientos de rectificación de área, linderos y medidas perimétricas antes indicados, pues la rectificación podría eventualmente afectar a los propietarios de los predios colindantes. Sin embargo, hay un gran porcentaje de predios en los cuales las discrepancias entre la descripción física real y registral se encuentran dentro de los rangos de las tolerancias catastrales registrales, pero, al no existir antecedentes gráficos en el Registro de Predios, no se puede incorporar el polígono del predio a la BGR o este se incorpora de manera inexacta. Por ende, cuando se solicite la inscripción de algún acto que modifique la descripción física del predio, se generan observaciones debido a la imposibilidad del Área de Catastro de verificar su adecuación con los antecedentes registrales, o por existir discrepancias con el polígono incorporado de manera inexacta a la BGR.

En estos casos, deberá prevalecer la información catastral para corregir las inexactitudes registrales, en atención a que el Registro de Predios no es un generador de catastro, sino que se nutre de la información que le proporcionan los entes generadores de catastro, quienes tienen la información oficial en cuanto a la determinación de la descripción física de un predio. Por tanto, la información registral incompleta o inexacta respecto a los datos descriptivos físicos del predio puede ser corregida por un título emitido por un ente generador de catastro, siempre que no se afecten los derechos de los propietarios colindantes.

La conclusión precedente ha sido abordada por dispositivos que regulan supuestos especiales de saneamiento de predios. Así, tenemos que, en el caso del saneamiento de predios rurales bajo competencia de los Gobiernos Regionales, el artículo 73 del Decreto Supremo N. ${ }^{\circ}$ 032-2008-VIVIENDA ${ }^{12}$ precisó que cuando en el Registro de Predios no exista información técnica suficiente, situación verificada por las áreas de catastro de las

12 Reglamento del Decreto Legislativo N. ${ }^{\circ} 1089$, decreto legislativo que establece el Régimen Temporal Extraordinario de Formalización y Titulación de Predios Rurales. 
zonas registrales, la información gráfica con que cuente el Organismo de Formalización de la Propiedad Informal (Cofopri) prevalece y sustituye a la que obra en el Registro de Predios; y, cuando corresponda, se extenderá el asiento de rectificación respectivo, siempre que no exceda los rangos de tolerancia permisibles.

Asimismo, en los supuestos de inscripción de predios en el marco de los proyectos de inversión pública y privada, el artículo 47 de la Ley $N .^{\circ} 30230^{13}$ establece la prevalencia de la información de campo sobre la información registral cuando (i) en el título archivado obran planos imperfectos que no cuentan con georreferenciación y/o área y/o medidas perimétricas, y/o no contengan datos técnicos, y/o estos contengan coordenadas arbitrarias; (ii) no existan planos como parte del título archivado, siempre que se encuentren dentro de los rangos de tolerancia respectivos; (iii) existan superposiciones gráficas generadas por desplazamiento de coordenadas o coordenadas en predios inscritos; (iv) el predio tenga discrepancias de cualquier dato técnico, dentro de los rangos de tolerancia establecidos en las normas del SNCP; y (v) la medida del área o medida perimétrica del predio inscrito esté expresada de forma distinta del sistema métrico decimal, siempre que la medida de esta última sea equivalente o esté dentro de los rangos de tolerancia respectivos.

Sin embargo, no existe una norma general que precise los supuestos de prevalencia de la información catastral emitida por un ente generador de catastro sobre la información registral inexacta o incompleta, lo que hace urgente modificar los alcances de la Ley N. ${ }^{\circ} 28294$ a fin de incorporar los supuestos en los que prevalecerá la información emitida por los entes generadores de catastro sobre la información registral incompleta o inexacta, y que sirva para corregir inexactitudes registrales.

Sin perjuicio de lo anterior, aun cuando no se modifique la Ley N. $^{\circ} 28294$, en nuestra opinión sí es posible aplicar en la calificación registral los supuestos de prevalencia de la información catastral sobre la información registral para corregir inexactitudes registrales, sobre la base de la naturaleza de la información de los entes generadores de catastro que, como señalamos anteriormente, tienen la versión oficial de las características físicas de un predio, siempre que no estemos ante los supuestos en que es necesario seguir alguno de los procedimientos de rectificación de área, linderos y medidas perimétricas.

Como ya se señaló, el TR ha admitido la prevalencia de la información catastral sobre la registral para corregir inexactitudes registrales en los supuestos de rectificación de área por error de cálculo y rectificación de área de manera unilateral, sin recurrir a alguno de los procedimientos de rectificación de área previstos en la normativa vigente.

13 Ley que establece medidas tributarias, simplificación de procedimientos y permisos para la promoción y dinamización de la inversión en el país. 
En efecto, en el Pleno XIX (2006), el TR aprobó el siguiente precedente de observancia obligatoria ${ }^{14}$ :

Es inscribible la rectificación del área de un predio urbano en mérito al plano y memoria descriptiva visados por la autoridad municipal correspondiente, prescindiendo de los mecanismos rectificatorios previstos por el artículo 13 de la Ley N. ${ }^{\circ}$ 27333, si el error surgió del equivocado o inexacto cálculo de su área, siempre que el Área de Catastro determine que los linderos, medidas perimétricas y ubicación espacial del predio no han sufrido variación alguna.

De acuerdo con este precedente, prevalecerá la información catastral emitida por un ente generador de catastro sobre la información registral inexacta para corregir el área de un predio, siempre que el Área de Catastro establezca que los linderos, medidas perimétricas y ubicación espacial del predio no han sufrido variación.

En la misma línea, mediante precedente aprobado en el Pleno CL-2016, el TR estableció el siguiente criterio vinculante:

Procede la rectificación de área, linderos y/o medidas perimétricas en mérito a escritura otorgada por el propietario acompañada de la documentación a que se refiere el artículo 20 del Reglamento de Inscripciones del Registro de Predios, tanto si se rectifica a área mayor o menor, siempre que la Oficina de Catastro determine indubitablemente que el polígono resultante se ubica dentro del ámbito gráfico del predio inscrito.

Por lo tanto, en este supuesto, se admite la inscripción de la rectificación de área del predio (para mayor o menor extensión), así como la de sus linderos y medidas perimétricas, siempre que el Área de Catastro determine indubitablemente que el polígono resultante se ubica dentro del ámbito gráfico del predio inscrito.

Desde luego, los criterios establecidos por el TR constituyen un avance para aceptar la prevalencia de la información gráfica validada por un ente generador de catastro sobre la información registral incompleta o inexacta, pero es insuficiente, pues respecto de un gran porcentaje de predios no existe información gráfica en los antecedentes registrales que permita determinar la concordancia entre la información catastral y la registral y, por tanto, no será posible aplicar los citados precedentes.

En otros supuestos, existe información gráfica incompleta o inexacta que ha conducido a que el polígono del predio sea incorporado de manera inexacta a la BGR, de tal manera que, cuando es contrastada con la información catastral, se encuentran desfases

14 Este precedente de observancia obligatoria fue precisado en los Plenos CXV y CLXXIV en los siguientes aspectos: (i) también se aplica para predios rurales; (ii) no se aplica cuando el Área de Catastro no puede determinar si los linderos, medidas perimétricas y ubicación espacial del predio han sufrido variación; y (iii) no se aplica para supuestos distintos al de error de cálculo. 
en cuanto a la ubicación espacial o discrepancias en cuanto al polígono, que impiden la aplicación de los precedentes citados.

Consideramos que, en los supuestos de inexistencia, deficiencia o error en la información gráfica que obra en los antecedentes registrales, deberá prevalecer la información técnica que ha sido validada por un ente generador de catastro y que consta en el título presentado, sin necesidad de recurrir a los procedimientos de rectificación de área, siempre que se cumplan los siguientes requisitos: (i) que exista concordancia entre la descripción literal del predio que consta en la partida registral y la que consta en el título presentado; y (ii) que, en caso de existir discrepancias, estas se encuentren dentro de los rangos de tolerancias catastrales y registrales.

\section{DEL ROL DE LAS ÁREAS DE CATASTRO Y LA VINCULATORIEDAD DE SUS INFORMES}

El segundo párrafo del artículo 11 del Reglamento de Inscripciones del Registro de Predios (RIRP) ha delimitado la actuación del Área de Catastro en el procedimiento registral, precisando que le corresponde: (i) verificar los datos técnicos del plano presentado; (ii) determinar la existencia o no de superposición de partidas, así como otros aspectos relevantes, si los hubiere; y (iii) elaborar un informe sobre la base de la información gráfica con la que cuente el Área de Catastro, actualizada a la fecha de emisión del informe técnico, bajo responsabilidad.

Uno de los mayores problemas que surgen con las decisiones del Área de Catastro se debe a que la verificación de la adecuación entre la descripción física de un predio que consta en el título presentado y el antecedente registral se efectúa sobre la base de la información gráfica con que cuenta el Registro de Predios, que en la mayoría de los casos es inexistente, incompleta o inexacta. Ello conduce a que el Área de Catastro no pueda determinar si un predio se encuentra o no inmatriculado, o que, en caso de estarlo, se pueda comprobar si existe o no superposición o adecuación con el predio descrito en el título objeto de solicitud de inscripción.

Parte del problema antes descrito ha sido superado en los casos de inmatriculación o de independización de predios, en los que se ha admitido la inscripción del título cuando el Área de Catastro no pueda determinar si un predio se encuentra o no inmatriculado, o si corre o no inscrito en la partida matriz de la que se solicita su independización, conforme a la previsión contenida en los artículos 16 y 59 del RIRP, respectivamente. Sin embargo, para los demás actos registrables que impliquen modificación de la descripción física del predio, la imposibilidad de determinar la adecuación de los datos descriptivos del predio con sus antecedentes registrales constituye un verdadero obstáculo para la inscripción del título, obstáculo que puede ser superado si se considera que la información catastral prevalece sobre la información registral inexistente, incompleta o inexacta, como sostenemos en el presente artículo. 
Otro de los aspectos que deben reevaluarse es la forma como se ha regulado en el RIRP los efectos del informe técnico del Área de Catastro. Así, se ha establecido en el tercer párrafo del artículo 11 del RIRP que "[e]l informe del Área de Catastro es vinculante para el registrador". Esta norma tiene como antecedente inmediato el precedente de observancia obligatoria aprobado en el X Pleno del TR, el cual establece lo siguiente:

El informe del área de catastro es vinculante para el registrador, siempre que se refiera a aspectos estrictamente técnicos. El registrador debe distinguir en su contenido los aspectos técnicos que sí lo vinculan, y otros aspectos de aplicación e interpretación de normas jurídicas, que no le competen a dicha área, sino de manera indelegable y exclusiva al registrador público.

De acuerdo con la definición de la Real Academia de la Lengua Española, el adjetivo vinculante significa "obligatorio", lo cual implica que el informe del Área de Catastro es obligatorio para las instancias de calificación registral, siempre que, como se indica en el precedente citado en el párrafo anterior, se refiera a aspectos estrictamente técnicos.

Sin embargo, desde el punto de vista jurídico, esta norma vulnera la competencia para la calificación, asignada por ley únicamente a las instancias de calificación registral, pues una parte de la calificación estará a cargo del Área de Catastro, sin que pueda ser cuestionada por dichas instancias, dado el carácter obligatorio de sus informes. Asimismo, desde el punto de vista práctico, lo que ocurre en la realidad es que todos los aspectos técnicos del informe del Área de Catastro no son revisados por las instancias de calificación registral, lo que causa que, aun en los supuestos de errores u omisiones, el contenido del informe quede convalidado.

En este sentido, es necesario que se modifique el artículo 11 del RIRP a fin de precisar que el informe del Área de Catastro es un acto previo para la calificación de los títulos que impliquen la incorporación de un predio al Registro de Predios o la modificación de su descripción física, pero que la opinión técnica debe ser evaluada por las instancias de calificación registral, quienes tienen la facultad de asumir dicha posición técnica o pedir las aclaraciones respectivas en caso de que detecten errores en ella. De lo contrario, el Área de Catastro se convierte en una suprainstancia de calificación de aspectos técnicos, con el riesgo de que sus decisiones erróneas no puedan ser revisadas por las instancias de calificación registral.

Creemos que el informe técnico del Área de Catastro sí tiene que ser vinculante, pero únicamente para dicha área, en el sentido de que la evaluación técnica efectuada no puede ser modificada en el mismo o en otro procedimiento administrativo, salvo que se haya variado la BGR por haberse inscrito un título posterior. 


\section{CONCLUSIONES}

1. En nuestro sistema registral, la mayoría de las inscripciones de las inmatriculaciones o modificaciones físicas de los predios se han efectuado sin tener soporte en información gráfica avalada por un ente generador de catastro, lo que ocasiona discrepancias entre la descripción registral del área, linderos y medidas perimétricas de los predios, y su descripción real.

2. Las discrepancias entre la descripción física de un predio conforme al Registro de Predios y la descripción física real ocasionan observaciones frecuentes cuando se solicita la inscripción de algún acto que implique la modificación de la descripción física del predio inscrito.

3. En los supuestos de inexistencia, deficiencia o error en la información gráfica que obra en los antecedentes registrales, debe prevalecer la información técnica que ha sido validada por un ente generador de catastro para corregir inexactitudes registrales sin recurrir a alguno de los procedimientos de rectificación de área, siempre que se cumplan los siguientes requisitos: (i) que exista concordancia entre la descripción literal del predio que consta en la partida registral y la que consta en documento gráfico avalado por un ente generador de catastro; y (ii) que, en caso de discrepancias en cuanto al área, linderos y medidas perimétricas, estas se encuentren dentro de los rangos de tolerancia.

4. Es necesario que se modifique la Ley N. ${ }^{\circ} 28294$, que crea el Sistema Nacional Integrado de Catastro, a fin de incorporar reglas que establezcan de manera general los supuestos en los que prevalecerá la información catastral sobre la información registral incompleta o inexacta, para corregir inexactitudes registrales.

5. En tanto no se modifique la Ley $\mathrm{N}^{\circ} 28294$, es posible aplicar en la calificación registral los supuestos de prevalencia de la información catastral sobre la registral para corregir las inexactitudes registrales, atendiendo a que la información gráfica avalada por un ente generador de catastro corresponde a la realidad física del terreno que no puede ser cuestionada por el Registro de Predios, porque este no genera catastro.

6. Es necesario modificar el artículo 11 del RIRP a fin de precisar que el informe del Área de Catastro no es vinculante (léase obligatorio) para las instancias de calificación registral, quienes tienen la facultad de asumir dicha posición técnica o de pedir las aclaraciones respectivas cuando detecten que es incorrecta. 
Prevalencia del catastro sobre el Registro de Predios para corregir inexactitudes registrales

\section{REFERENCIAS}

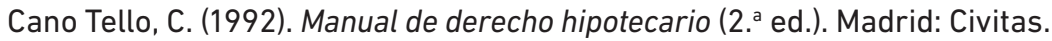

Real Academia Española. (2020). Diccionario de la lengua española. Recuperado de https:// dle.rae.es/vinculante 
